\title{
Circulating endothelial cells and other angiogenesis factors in pancreatic carcinoma patients receiving gemcitabine chemotherapy
}

Shunsuke Kondo ${ }^{1 *}$, Hideki Ueno ${ }^{1}$, Jun Hashimoto ${ }^{1}$, Chigusa Morizane ${ }^{1}$, Fumiaki Koizumi ${ }^{2}$, Takuji Okusaka ${ }^{1}$ and Kenji Tamura ${ }^{3}$

\begin{abstract}
Background: Pancreatic carcinoma is a significant cause of cancer-related death in developed countries. As the level of circulating endothelial cells (CECs) is known to increase in response to various cancers, we investigated the predictive potential of CEC levels and the association of these levels with the expression of proangiogenic factors in pancreatic carcinoma patients.

Methods: Pancreatic carcinoma patients receiving gemcitabine chemotherapy were prospectively assigned to this study. CEC levels were measured using the CellTracks system, and the plasma levels of several angiogenesis factors were measured using multiplex immunoassay. Associations between clinical outcomes and the levels of these factors were evaluated.

Results: Baseline CEC levels were markedly higher in pancreatic carcinoma patients $(n=37)$ than in healthy volunteers $(n=53)$. Moreover, these high CEC levels were associated with decreased overall survival (median, 297 days versus 143 days, $P<0.001$ ) and progression-free survival (median, 150 days versus 64 days, $P=0.008$ ), as well as with high vascular endothelial growth factor, interleukin (IL)-8, and IL-10 expression in the pancreatic carcinoma patients.
\end{abstract}

Conclusions: Several chemokines and proangiogenic factors correlate with the release of CECS, and the number of CECs detected may be a useful prognostic marker in pancreatic carcinoma patients undergoing gemcitabine chemotherapy.

Trial registration: UMIN000002323

Keywords: Pancreatic carcinoma, Circulating endothelial cells, Angiogenesis factors

\section{Background}

Pancreatic carcinoma is one of the most lethal tumors and is the fourth leading cause of cancer-related death in developed nations [1]. As pancreatic carcinoma has a high propensity for both local invasion and distant metastasis, surgery is precluded as a treatment for most patients who present with advanced-stage disease. These patients have a median survival of only 6 months and an overall 5-year survival of less than 5\%. The prognosis for advanced pancreatic carcinoma patients is therefore

\footnotetext{
* Correspondence: shkondo@ncc.go.jp

'Hepatobiliary and Pancreatic Oncology Division, National Cancer Center Hospital, Tokyo, Japan

Full list of author information is available at the end of the article
}

extremely poor, and the impact of standard therapy is only modest, despite many advances that have improved the outcome of this disease.

Pancreatic carcinoma is not a grossly vascular tumor; however, it overexpresses multiple mitogenic growth factors that are also angiogenic, such as epidermal growth factor (EGF), hepatocyte growth factor (HGF), fibroblast growth factor (FGF), platelet-derived growth factor $\mathrm{B}$ chain (PDGF-BB), and vascular endothelial growth factor (VEGF). Angiogenesis often occurs in response to an imbalance in which proangiogenic factors predominate over antiangiogenic factors. For instance, VEGF expression has been shown to promote tumor growth in pancreatic carcinomas [2]. High VEGF expression is also

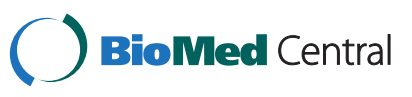


associated with increased microvessel density [3] and is a predictor of poor outcomes and early tumor recurrence after curative resection [4]. Although agents that target the VEGF signaling pathway have been shown to inhibit tumor growth, metastasis, and angiogenesis [5], treating advanced pancreatic carcinoma patients with axitinib-a selective inhibitor of VEGF receptors 1, 2, and 3-in combination with gemcitabine was not found to improve overall survival in a phase 3 trial [6]. Despite this finding, proangiogenic factors remain an important therapeutic target for the treatment of pancreatic carcinoma.

Circulating endothelial cells (CECs) are mature cells that are not associated with vessel walls but are detached from the endothelium and circulate within peripheral blood. The number of CECs present in the blood has been found to increase in response to cardiovascular disease, vasculitis, infectious disease, and various cancers $[7,8]$. Indeed, the level of CECs has been recognized as a useful biomarker for vascular damage. It has also been reported that the number of CECs found in non-small cell lung cancer patients treated with carboplatin plus paclitaxel is a promising predictive marker of the clinical efficacy of these drugs [9]. We believe that CEC levels may also be a potential biomarker for pancreatic carcinoma; therefore, we investigated the levels of CECs found in patients with different severities of pancreatic carcinoma, as well as the effects of gemcitabine treatment on CEC levels. Furthermore, the associations between CEC levels and the expression levels of several factors involved in angiogenesis and neovascularization were also examined in this study.

\section{Methods}

\section{Study approval}

This prospective study was approved by the Institutional Review Board of the National Cancer Center, and written informed consent was obtained from all patients. This study is registered with the University Hospital Medical Information Network in Japan (UMIN; number UMIN000002323) and has been completed.

\section{Patients and blood sample collection}

A total of 37 chemotherapy-naive patients with histologically or cytologically confirmed invasive ductal pancreatic carcinoma were prospectively enrolled in this study between April 2009 and March 2010 and received gemcitabine chemotherapy. Patients with coexisting infections and/or cardiovascular illness were excluded. The detailed history of all the patients was obtained and a physical examination was performed before beginning gemcitabine treatment. Pretreatment baseline laboratory parameters were also assessed for all patients. The baseline tumor status of each patient was evaluated using computed tomography (CT) scans of the chest, abdomen, and pelvis, while peripheral blood sampling was performed both prior to treatment initiation (baseline) and at day $28 \pm 7$ after starting chemotherapy. A dose of $1000 \mathrm{mg} / \mathrm{m}^{2}$ gemcitabine was administered intravenously for $30 \mathrm{~min}$ on days 1,8 , and 15 of a 28-day cycle until disease progression, unacceptable toxicity, or patient refusal occurred. The data collected included those pertaining to standard demographics and disease characteristics, the date of initial treatment, the best response to treatment, date of progression, and the date of death or last follow-up. The tumors were evaluated every 68 weeks after starting each course of gemcitabine, and best responses were documented according to the $\mathrm{Re}$ sponse Evaluation Criteria in Solid Tumors (RECIST).

\section{CEC enumeration}

Blood samples from advanced pancreatic carcinoma patients were drawn into $10 \mathrm{~mL}$ CellSave Preservative Tubes (Immunicon Corp. Huntingdon Valley, PA) for CEC enumeration. Samples were obtained both before starting chemotherapy (baseline) and at $28 \pm 7$ days after starting chemotherapy. Samples were kept at room temperature and processed within $42 \mathrm{~h}$ of collection. All of the evaluations were performed without knowledge of the clinical status of the patients. The CellTracks system (Veridex, LLC), which consists of the CellTracks AutoPrep system and the CellSpotter Analyzer system, was used for endothelial cell enumeration. In this system, CECs are defined as $\mathrm{CD}_{146} / \mathrm{DAPI}^{+} / \mathrm{CD} 105-\mathrm{PE}^{+} / \mathrm{CD} 45 \mathrm{APC}^{-}$ cells. Briefly, $\mathrm{CD} 146^{+}$cells were captured immunomagnetically by using ferrofluids coated with CD146 antibodies. The enriched cells were then labeled with the nuclear dye 4 V, 6-diamidino-2-phenylindole (DAPI), CD105 antibodies were conjugated to phycoerythrin (CD105-PE), and the pan-leukocyte antibody CD45 was conjugated to allophycocyanin (CD45-APC). Cells with the $\mathrm{DAPI}^{+} / \mathrm{CD} 105^{+} / \mathrm{CD} 45^{-}$phenotype were enumerated. We evaluated morphological cell viability and excluded dead cells from the cell count. The number of CECs in each sample was determined twice, and the mean value was calculated.

\section{Antibody suspension bead array system}

Peripheral blood was drawn into prechilled tubes containing ethylenediaminetetraacetic acid; was immediately subjected to centrifugation at $1000 \mathrm{~g}$ and $4^{\circ} \mathrm{C}$ for $15 \mathrm{~min}$, plasma was transferred to microtubes and subjected to further centrifugation at $10,000 \mathrm{~g}$ and $4^{\circ} \mathrm{C}$ for $10 \mathrm{~min}$ to remove contaminating platelets. Plasma samples were collected from patients before gemcitabine treatment was initiated and were stored at $-80^{\circ} \mathrm{C}$ until they were used for testing. The plasma concentrations of 7 biological markers (interleukin [IL]-6, IL-8, IL-10, 
PDGF-BB, VEGF, HGF, and SDF-1 alpha) were assayed in a subgroup of patients and control individuals by using the Bio-Plex suspension array system (Bio-Rad, Hercules, CA), which allows the simultaneous identification of cytokines in a 96-well filter plate. In brief, the appropriate cytokine standards and diluted plasma samples were added to a 96-well filter plate and incubated at room temperature for $30 \mathrm{~min}$ with antibodies chemically attached to fluorescent-labeled micro beads. After 3 filter washes, premixed detection antibodies were added to each well and incubated for $30 \mathrm{~min}$. After 3 more washes, premixed streptavidin-phycoerythrin was added to each well and incubated for $10 \mathrm{~min}$, followed by 3 more washes. The beads were then resuspended in
$125 \mu \mathrm{L}$ of assay buffer and the reaction mixture was quantified using the Bio-Plex protein array reader. Data were automatically processed and analyzed with BioPlex Manager Software 4.1 by using the standard curve obtained using a recombinant cytokine standard.

\section{Statistical analyses}

The Mann-Whitney test was used to compare the distributions of clinical factors and marker concentrations between patients with progressive disease (PD) and those without PD, stages III and IV disease, or recurrence. The survival time (progression-free survival [PFS] and overall survival [OS]) and clinical factors (age, gender, and Eastern Cooperative Oncology Group [ECOG] performance status

Table 1 Patient characteristics and CEC detection

\begin{tabular}{|c|c|c|c|c|c|}
\hline & & Mean CEC level 166 cells $/ 4 \mathrm{~mL}$ & Range (2-1195 cells/4 mL) & Total & $P^{a}$ \\
\hline & & $\geq 166$ cells $/ 4 \mathrm{~mL}$ & $<166$ cells $/ 4 \mathrm{~mL}$ & & \\
\hline & & CEC high & CEC $^{\text {low }}$ & & \\
\hline & & 12 & 25 & 37 & \\
\hline \multirow[t]{2}{*}{ Age } & Over 70 & 8 & 10 & $18(49 \%)$ & 0.17 \\
\hline & Below 70 & 4 & 15 & $19(51 \%)$ & \\
\hline \multirow[t]{2}{*}{ Sex } & Male & 7 & 17 & $24(65 \%)$ & 0.72 \\
\hline & Female & 5 & 8 & $13(35 \%)$ & \\
\hline \multirow[t]{3}{*}{ Stage } & III & 3 & 11 & $14(38 \%)$ & 0.59 \\
\hline & IV & 8 & 12 & $20(54 \%)$ & \\
\hline & Recurrence & 1 & 2 & $3(8 \%)$ & \\
\hline \multirow[t]{3}{*}{ ECOG PS } & 0 & 5 & 18 & $23(62 \%)$ & 0.09 \\
\hline & 1 & 6 & 4 & $10(27 \%)$ & \\
\hline & 2 & 1 & 3 & $4(11 \%)$ & \\
\hline \multirow[t]{3}{*}{ Pancreatic tumor location } & Head & 5 & 12 & $17(46 \%)$ & $>0.9$ \\
\hline & Body & 5 & 9 & $14(38 \%)$ & \\
\hline & Tail & 2 & 4 & $6(16 \%)$ & \\
\hline \multirow[t]{2}{*}{ CA19-9 (U/mL) } & $\geq 10,000$ & 3 & 5 & $8(22 \%)$ & $>0.9$ \\
\hline & $<10,000$ & 9 & 20 & $29(78 \%)$ & \\
\hline \multirow[t]{2}{*}{ CRP (mg/dL) } & $\geq 1.0$ & 7 & 3 & $10(27 \%)$ & $<0.01$ \\
\hline & $<1.0$ & 5 & 22 & $27(73 \%)$ & \\
\hline \multirow[t]{4}{*}{ Histology } & Poorly differentiated & 5 & 9 & $14(38 \%)$ & 0.62 \\
\hline & Moderately differentiated & 4 & 10 & $14(38 \%)$ & \\
\hline & Adenosquamous & 1 & 0 & $1(2 \%)$ & \\
\hline & N.E (cytology only) & 2 & 6 & $8(22 \%)$ & \\
\hline \multirow[t]{3}{*}{ Tumor response } & Partial response & 2 & 2 & $4(11 \%)$ & $<0.05$ \\
\hline & Stable disease & 4 & 18 & $22(59 \%)$ & \\
\hline & Progressive disease & 6 & 5 & $11(30 \%)$ & \\
\hline \multirow[t]{3}{*}{ Second line therapy } & S-1 & 6 & 12 & $18(49 \%)$ & 1 \\
\hline & Oxaliplatin + S-1 & 0 & 2 & $2(5 \%)$ & \\
\hline & No & 6 & 11 & 17 (46\%) & \\
\hline
\end{tabular}

${ }^{a} P$ values were calculated for each variable using Fisher's exact test.

Abbreviations: $\mathrm{CEC}=$ circulating endothelial cell; ECOG = Eastern Cooperative Oncology Group; CA19-9= carbohydrate antigen 19-9; CRP=C-reactive protein. 

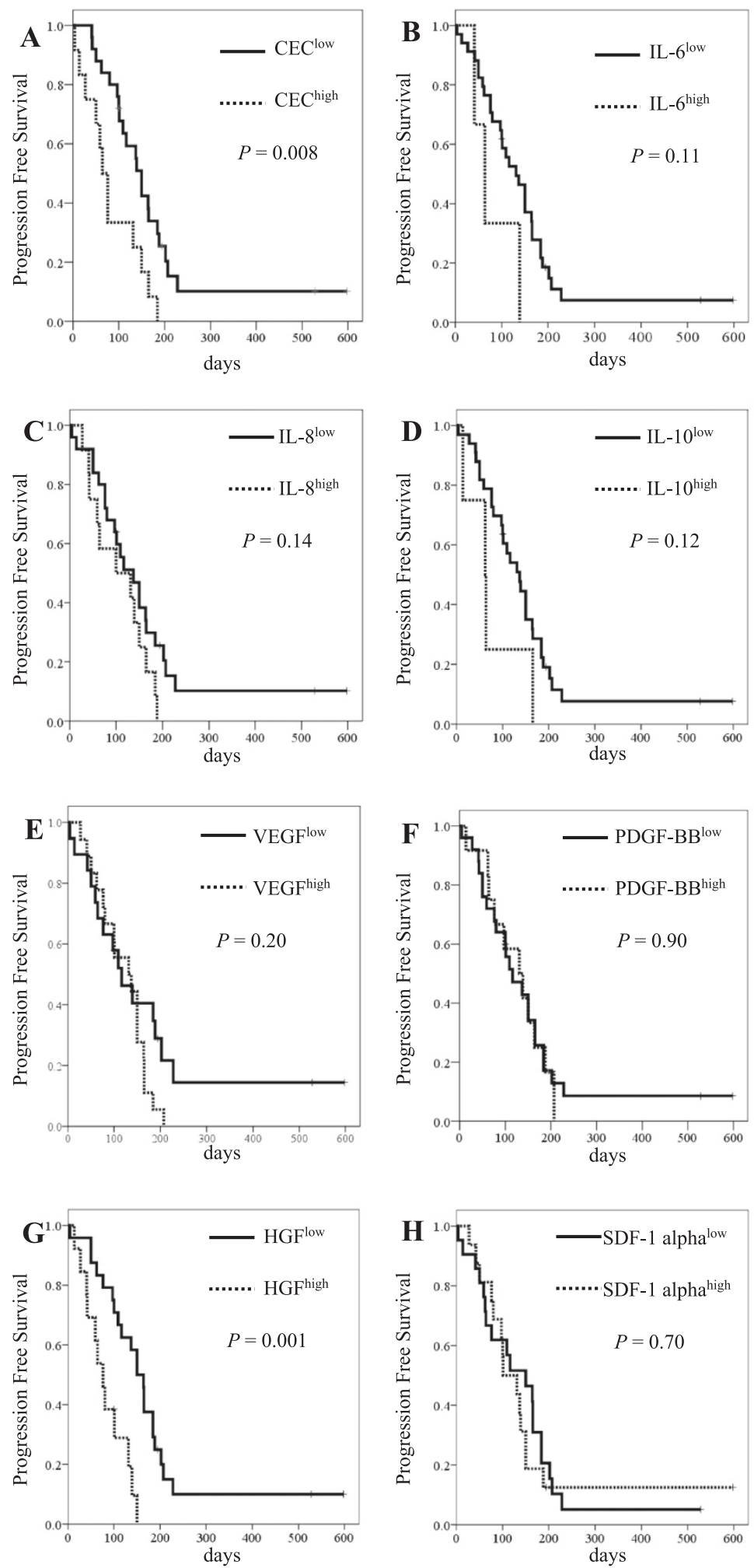

Figure 1 Kaplan-Meier curves for (A) progression-free survival with CEC counts, (B) progression-free survival with IL-6 levels, (C) progression-free survival with IL-8 levels, (D) progression-free survival with IL-10 levels, (E) progression-free survival with VEGF levels, (F) progression-free survival with PDGF-BB levels, (G) progression-free survival with HGF levels, and (H) progression-free survival with SDF-1 alpha levels. The cut-off points for the angiogenic factors were determined to be equal to or greater than these mean levels. 
[PS], and clinical stage of the patients) were examined using the Cox proportional hazards model. The survival curves for PFS and OS were estimated using the KaplanMeier method. Kaplan-Meier curves were used only to determine the trends of the associations between the molecules and PFS/OS, as any determination of the optimal cutoff point for the molecules relative to PFS/OS was beyond the scope of the present study. All statistical analyses were performed using IBM SPSS Statistics 18 (IBM Corporation, Somers, NY, USA).

\section{Results}

\section{Patient characteristics}

A total of 37 patients with pancreatic carcinoma were prospectively enrolled in this study. Fourteen of these patients (38\%) presented with locally advanced pancreatic carcinoma, 20 patients $(54 \%)$ presented with metastases, and 3 patients (8\%) were enrolled following recurrence after surgery. Twenty-three patients (62\%) had ECOG PS0, 10 patients (27\%) had ECOG PS1, and 4 patients (11\%) had ECOG PS2. Histologically, 14 patients (38\%) had poorly differentiated adenocarcinoma, 14 patients (38\%) had moderately differentiated adenocarcinoma, 1 patient (2\%) had an adenosquamous tumor, and 8 patients (22\%) had cytological adenocarcinoma. No patient experienced a complete response to treatment. Four patients (11\%) exhibited a partial response (PR) rate to treatment (11\%), stable disease (SD) was observed in 22 patients (59\%), and PD was observed in 11 patients (30\%). Second-line therapy was administered to 20 patients (54\%), whereby 18 patients (49\%) received S-1 monotherapy and 2 patients $(5 \%)$ received oxaliplatin and S-1 combination therapy (Table 1).

\section{Baseline levels of CECs and angiogenic factors}

The mean CEC level found in the pancreatic carcinoma patients was 166 cells/4 mL (range: $2-1195$ cells/4 mL) while the median CEC level was 66 cells $/ 4 \mathrm{~mL}$. These CEC levels were higher than those of randomly-selected healthy volunteers $(P<0.01)$, as previously reported $(\mathrm{n}=53$, mean $\pm \mathrm{SD}=46.2 \pm 86.3$ cells $/ 4 \mathrm{~mL})$ [9]. In this study, the cut-off point of $\mathrm{CEC}^{\text {high }}$ was determined to be equal to or greater than 166 cells $/ 4 \mathrm{~mL}$ while that of $\mathrm{CEC}^{\text {low }}$ was lower than 166 cells $/ 4 \mathrm{~mL}$. CEC ${ }^{\text {high }}$ was significantly associated with high levels of $\mathrm{C}$-reactive protein (CRP) (over $1.0 \mathrm{mg} / \mathrm{dL} ; P<0.01$ ). The median PFS was 64 days (95\% confidence interval [CI], 45-83) in the $\mathrm{CEC}^{\text {high }}$ group, while that in the $\mathrm{CEC}^{\text {low }}$ group was 150 days (95\% CI, 130-170; log-rank test; $P=0.008$; Figure 1A). The median OS was 143 days (95\% CI, 53-233) in the $\mathrm{CEC}^{\text {high }}$ group and 297 days (95\% CI, 240-354) in the $\mathrm{CEC}^{\text {low }}$ group (log-rank test; $P<0.001$; Figure 2A). Univariate analysis of CEC levels and clinical factors for OS was performed using the Cox proportional hazard model. The hazard ratio (HR) for CEC levels $\left(\mathrm{CEC}^{\text {high }}\right.$ versus $\mathrm{CEC}^{\text {low }}$ ) was 5.18 (95\% CI, 2.23-12.03; $P<0.001)$.

The mean levels of IL-6, IL-8, IL-10, PDGF-BB, VEGF, HGF, and SDF-1 alpha were found to be $19.3 \mathrm{pg} / \mathrm{mL}, 11.3 \mathrm{pg} / \mathrm{mL}, 7.82 \mathrm{pg} / \mathrm{mL}, 1127.5 \mathrm{pg} / \mathrm{mL}$, $44.1 \mathrm{pg} / \mathrm{mL}, 471.3 \mathrm{pg} / \mathrm{mL}$, and $110.6 \mathrm{pg} / \mathrm{mL}$, respectively. The cut-off points for the angiogenic factors were determined to be equal to or greater than these mean levels, and the median PFS in $\mathrm{HGF}^{\text {low }}$ was longer than the $\mathrm{HGF}^{\text {high }}$ group $(P=0.001$; Figure $1 \mathrm{G})$. However, other factors were not found to have statistical significance with regard to PFS. The median OS was longer in the case of IL-10 (112 days [95\% CI, 50-173] in IL-10 ${ }^{\text {high }}$ vs. 264 days [95\% CI, 204-324] IL-10 ${ }^{\text {low }}$, log-rank test: $P=0.003$; Figure 2d) and HGF (150 days [95\% CI, 65234] in $\mathrm{HGF}^{\text {high }}$ vs. 291 days [95\% CI, 223-359] in HGF $^{\text {low }}$, log-rank test: $P=0.01$; Figure $2 \mathrm{G}$ ).

Among the clinical factors that were examined in this study, a poor PS (PS 1 and 2), advanced stage (stage IV and recurrence), and high levels of IL-10, HGF, and CRP were significantly correlated with poor $\mathrm{OS}$ in univariate cox analysis, with $\mathrm{HRs}$ of 2.72 (95\% CI, 1.29-5.70; $P=0.008), 2.21$ (95\% CI, 1.03-4.71; $P=0.04), 5.05$ (95\% CI, 1.55-16.39; $P=0.007), 2.52$ (95\% CI, 1.22-5.21; $P=0.01$ ), and 2.49 (95\% CI, 1.14-5.42; $P=0.02$ ), respectively. In a multivariate Cox analysis model that included clinical stage, PS, CRP levels, CEC levels, IL-10 levels, and HGF levels, the number of CECs detected remained statistically stable at 0.05 . The resulting $\mathrm{HRs}$ were 2.04 (95\% CI, 0.78-5.35; $P=0.15$ ), 2.58 (95\% CI, 0.98-6.76; $P>0.05$ ), 2.04 (95\% CI, 0.62-6.76; $P=0.24$ ), 5.14 (95\% CI, $1.83-14.45, P=0.002), 5.26$ (95\% CI, 1.26-22.22; $P=0.02$ ) and $1.34 \quad(95 \% \mathrm{CI}, \quad 0.46-3.91 ; P=0.59)$, respectively (Table 2).

\section{Changes in CEC number during treatment}

The number of CECs was analyzed in 22 of the 37 patients at $28 \pm 7$ days after the start of gemcitabine therapy. The mean number of CECs detected in these patients after $28 \pm 7$ days was 133 cells $/ 4 \mathrm{~mL}$ (range: 15-664 cells $/ 4 \mathrm{~mL}$ ), while the median number of CECs was 68 cells $/ 4 \mathrm{~mL}$. The absolute counts of CECs did not change significantly between day 1 and day $28 \pm 7$ of treatment (Mann-Whitney test, $P=0.11$ ). Furthermore, a change in CEC counts from baseline to after $28 \pm 7$ days of treatment was not statistically associated with tumor response (Mann-Whitney test, $P>0.05$, Figure 3).

\section{Association between CEC number and blood angiogenic factors}

The numbers of CECs were compared between nonPD (PR and SD, $n=26)$ and PD patients $(n=11)$ for 

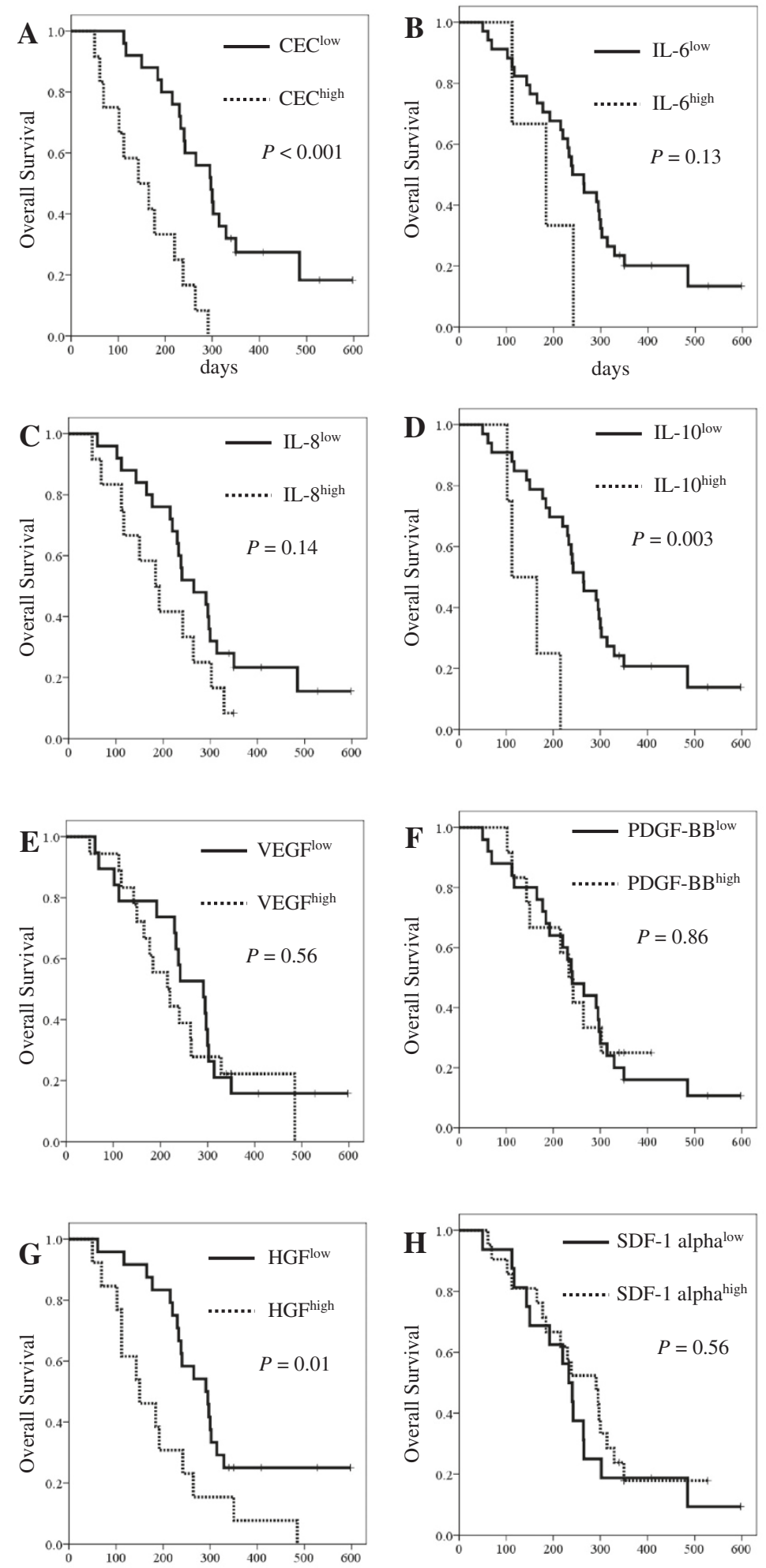

Figure 2 Kaplan-Meier curves for (A) overall survival with CEC counts, (B) overall survival with IL-6 levels, (C) overall survival with IL-8 levels, (D) overall survival with IL-10 levels, (E) overall survival with VEGF levels, (F) overall survival with PDGF-BB levels, (G) overall survival with HGF levels, and $(\mathrm{H})$ overall survival with SDF-1 alpha levels. The cut-off points for the angiogenic factors were determined to be equal to or greater than these mean levels. 
all markers. The baseline levels of CEC ( $P=0.03)$, IL-6 $(P<0.01)$, and IL-10 $(P=0.03)$ were found to be significantly higher among patients with $\mathrm{PD}$ than among those with PR or SD. The blood concentrations of HGF $(P<0.001)$, IL-6 $(P<0.01)$, and IL-8 $(P<0.001)$ were also significantly higher among patients with clinical stage IV disease and recurrence than among those with stage III disease. When the association between CEC number and the expression of other angiogenic factors was examined, the number of CECs was found to correlate positively with the levels of VEGF $(\mathrm{r}=0.34, P=0.04)$, HGF $(\mathrm{r}=0.37$, $P=0.02)$, IL-8 $(\mathrm{r}=0.38, P=0.02)$, and IL-10 $(\mathrm{r}=0.45$, $P=0.006)$, suggesting that the number of CECs is related to the expression of these markers (Table 3).

\section{Discussions}

In most cases, CECs are apoptotic or necrotic cells that are released into circulation as a byproduct of vascular turnover. In some cancer patients, the level of CECs is significantly higher than that of healthy individuals, and this increased level has been identified as a surrogate marker of angiogenesis and anti-angiogenic drug activity $[10,11]$. The present study has shown that baseline CEC levels are markedly higher among pancreatic carcinoma patients than in healthy individuals. Our results also support the hypothesis that CEC levels are associated with clinical outcome in pancreatic carcinoma patients undergoing gemcitabine chemotherapy, and may be a prognostic factor for this disease. A previous study found that the baseline level of CECs, identified as $\mathrm{CD} 45^{-} \mathrm{CD} 31^{+} \mathrm{CD} 34^{+}$by flow cytometry, was inversely associated with OS in patients who had gemcitabinerefractory metastatic pancreatic carcinoma and were treated with bevacizumab plus erlotinib [12]. CEC $\left(\mathrm{CD} 45^{-} \mathrm{CD} 31^{+} \mathrm{CD} 146^{+}\right)$detection by flow cytometry requires careful discrimination between blood cell populations with overlapping phenotypes showing hallmarks of $\mathrm{T}$ cells $\left(\mathrm{CD} 45^{-} \mathrm{CD} 31^{-} \mathrm{CD} 146^{+}\right)$and platelets $\left(\mathrm{CD} 45^{-}\right.$ $\left.\mathrm{CD} 31^{\text {high }} \mathrm{CD} 146^{-}\right)$. These cells populations show distinct regulation during cancer therapy, and their concomitant analysis may offer extended prognostic and predictive information [13].

Table 2 Univariate and multivariate Cox analyses of prognosis

\begin{tabular}{|c|c|c|c|}
\hline Univariate analysis & HR & $95 \% \mathrm{Cl}$ & $P$ \\
\hline Age: Over 70 vs. Below 70 & 0.52 & $0.25-1.13$ & 0.1 \\
\hline Sex: Male vs. Female & 1.00 & $0.48-2.08$ & 0.99 \\
\hline Stage: IV + Recurrence vs. III & 2.21 & $1.03-4.71$ & 0.04 \\
\hline ECOG PS: $2+1$ vs. 0 & 2.72 & $1.29-5.70$ & 0.008 \\
\hline Pancreatic tumor location: Head vs. Others & 0.94 & $0.46-1.90$ & 0.86 \\
\hline CA19-9 (cut-off: 10,000 U/mL): CA19-9 high vs. CA19-9 $9^{\text {low }}$ & 1.77 & $0.75-4.15$ & 0.19 \\
\hline CRP level (cut-off: $1.0 \mathrm{mg} / \mathrm{dL}$ ): CRP high vs. CRP low & 2.49 & $1.14-5.42$ & 0.02 \\
\hline Histology: Poorly differentiated vs. Others & 1.09 & $0.52-2.27$ & 0.82 \\
\hline Second line therapy: Yes vs. No & 0.61 & $0.30-1.24$ & 0.17 \\
\hline CEC level (cut-off: 166 cells/4 mL): CEChigh vs. CEC low & 5.18 & $2.23-12.03$ & $<0.001$ \\
\hline IL-6 (cut-off: $19.3 \mathrm{pg} / \mathrm{mL}$ ): IL-6 high vs. IL-6 low & 2.52 & $0.73-8.64$ & 0.14 \\
\hline IL-8 (cut-off: 11.3 pg/mL): IL-8 high vs. IL-8 low & 1.74 & $0.82-3.67$ & 0.15 \\
\hline IL-10 (cut-off: 7.82 pg/mL): IL-10 high vs. IL-10 low & 5.05 & $1.55-16.39$ & 0.007 \\
\hline VEGF (cut-off: $44.1 \mathrm{pg} / \mathrm{mL}$ ): VEGF high vs. VEGF & 1.22 & $0.60-2.47$ & 0.59 \\
\hline PDGF-BB (cut-off: 1127.5 pg/mL): PDGF-BB high vs. PDGF-BB low & 0.93 & $0.43-2.04$ & 0.86 \\
\hline HGF (cut-off: 471.3 pg/mL): HGF high vs. HGF ${ }^{\text {low }}$ & 2.52 & $1.22-5.21$ & 0.01 \\
\hline SDF-1 alpha (cut-off: 110.6 pg/mL): SDF-1 alpha high vs. SDF-1 alphalow & 1.23 & $0.60-2.53$ & 0.56 \\
\hline Multivariate analysis & HR & $95 \% \mathrm{Cl}$ & $P$ \\
\hline Stage: IV + Recurrence vs. III & 2.04 & $0.78-5.35$ & 0.15 \\
\hline ECOG PS: $2+1$ vs. 0 & 2.58 & $0.98-6.76$ & $>0.05$ \\
\hline CRP level (cut-off: $1.0 \mathrm{mg} / \mathrm{dL}$ ): CRP high vs. CRP low & 2.04 & $0.62-6.76$ & 0.24 \\
\hline CEC level (cut-off: 166 cells/4 mL): CEChigh vs. CEClow & 5.14 & $1.83-14.45$ & 0.002 \\
\hline IL-10 (cut-off: 7.82 pg/mL): IL-10 high vs. IL-10 low & 5.26 & $1.26-22.22$ & 0.02 \\
\hline HGF (cut-off: 471.3 pg/mL): HGF high vs. HGF ${ }^{\text {low }}$ & 1.34 & $0.46-3.91$ & 0.59 \\
\hline
\end{tabular}

Abbreviations: $\mathrm{HR}=$ hazard ratio; $\mathrm{Cl}=$ confidence interval; $\mathrm{ECOG} \mathrm{PS}=$ Eastern Cooperative Oncology Group performance status; $\mathrm{CEC}=$ circulating endothelial cells; $\mathrm{IL}=$ interleukin; PDGF-BB = platelet-derived growth factor-B chain; VEGF = vascular endothelial growth factor; $\mathrm{HGF}=$ hepatocyte growth factor; $C A 19-9$ $=$ carbohydrate antigen $19-9 ; \mathrm{CRP}=\mathrm{C}$-reactive protein; $\mathrm{CEA}=$ carcinoembryonic antigen. 


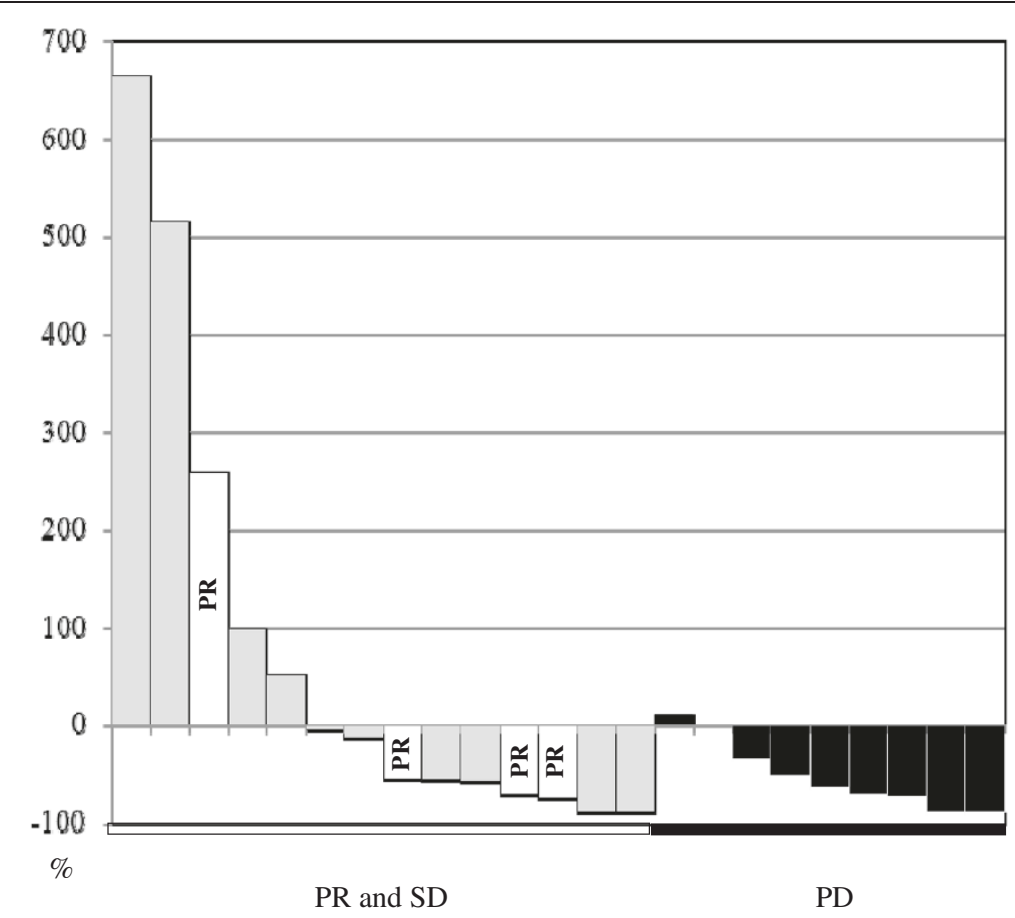

Figure 3 Waterfall plot showing the changes in CEC counts and tumor response in patients without progressive disease (PD) (those with partial response [PR] or stable disease [SD]) and patients with PD, after $28 \pm 7$ days of gemcitabine treatment.

Our study also found the baseline level of CECs, as well as the levels of HGF, IL-6, and IL-10, which are associated with gemcitabine resistance or stemness, to be significantly higher among PD patients. Univariate Cox model analysis further demonstrated that PS, clinical stage, CRP levels, and CEC levels are all associated with the survival of pancreatic carcinoma patients, while multivariate Cox analysis showed that CEC and IL-10 levels are strongly associated with survival.
The number of CECs detectable in individuals has previously been found to be associated with the plasma levels of VCAM-1 and VEGF in cancer patients [14] [15]. Our findings further show that, in addition to VEGF, CEC levels are strongly associated with the expression levels of IL-8, IL-10, and HGF in pancreatic carcinoma patients. These molecules, among others, play important roles in tumor biology and have been implicated in several cellular phenotypes. Chemokines,

Table 3 Association between CECs and other factors

\begin{tabular}{|c|c|c|c|}
\hline & Mean \pm SD & Spearman's rank correlation coefficient & $P$ \\
\hline CEC (cells/4 mL) & $166.2 \pm 228.9$ & 1 & - \\
\hline IL-6 (pg/mL) & $19.3 \pm 52.4$ & 0.17 & 0.30 \\
\hline IL-8 (pg/mL) & $11.3 \pm 10.1$ & 0.38 & 0.02 \\
\hline IL-10 (pg/mL) & $7.82 \pm 26.9$ & 0.45 & 0.006 \\
\hline VEGF $(p g / m L)$ & $44.1 \pm 38.8$ & 0.34 & 0.04 \\
\hline PDGF-BB (pg/mL) & $1,127.5 \pm 941.5$ & 0.24 & 0.16 \\
\hline $\mathrm{HGF}(\mathrm{pg} / \mathrm{mL})$ & $471.3 \pm 249.0$ & 0.37 & 0.02 \\
\hline SDF-1alpha (pg/mL) & $110.6 \pm 43.7$ & 0.15 & 0.37 \\
\hline $\mathrm{CRP}(\mathrm{mg} / \mathrm{dL})$ & $1.9 \pm 3.9$ & 0.31 & 0.06 \\
\hline CA19-9 (U/mL) & $18,229.1 \pm 55,377.8$ & 0.11 & 0.50 \\
\hline CEA (ng/mL) & $18.3 \pm 51.0$ & 0.03 & 0.88 \\
\hline
\end{tabular}

Abbreviations: $\mathrm{CEC}=$ Circulating endothelial cell; IL = interleukin; PDGF-BB = platelet-derived growth factor-B chain; VEGF = vascular endothelial growth factor; $\mathrm{HGF}=$ hepatocyte growth factor; $\mathrm{CA19-9}=$ carbohydrate antigen 19-9; CRP =C-reactive protein; $\mathrm{CEA}=$ carcinoembryonic antigen. 
including IL-8 and IL-10, are small peptides involved in controlling cell migration, particularly in leukocytes, during inflammation and the immune response. Chemokines are also important in tumor biology as they influence tumor growth, invasion, metastasis, and angiogenesis. For instance, VEGF, HGF and IL-8 significantly stimulate the proliferation, migration, and invasion of cancer cells. CEC are shed from vessels and this process may be amplified by an aberrant vascular turnover/remodeling associated with high local levels of VEGF required for CEC survival [16]. The chemokine SDF-1 has likewise been found to enhance the production of IL-8 by pancreatic cells in a paracrine manner [17]. Although our results did not indicate that SDF-1 levels were associated with CEC or IL-8 levels in the pancreatic cancer patients examined, it is likely that several of the proangiogenic factors examined in this study interact with each other to promote vascular turnover and remodeling, thereby leading to a higher number of CECs in the peripheral blood of cancer patients.

Drugs targeting angiogenesis, such as those that inhibit the VEGF pathway, have had a major impact in the treatment of many types of cancer. The VEGF pathway is also an independent prognostic factor for patient survival in pancreatic carcinoma. Although preclinical models have suggested that VEGF-VEGF receptor inhibitors would be effective in the treatment of pancreatic carcinoma, patients who received bevacizumab and axitinib therapy in addition to gemcitabine have not shown a survival advantage when compared to those treated with gemcitabine alone $[6,18]$. These results add to the increasing evidence that suggests that targeting VEGF signaling is an ineffective strategy in the treatment of pancreatic carcinoma. However, many antiangiogenic therapies modulate the expression levels of proangiogenic factors [19], and many factors are associated with tumor angiogenesis. Therefore, there are a variety of potential therapeutic targets that may be exploited in order to target angiogenesis, potentially including those examined in this study.

In advanced non-small cell lung cancer (NSCLC), patients with higher baseline CEC counts have PR/SD and longer PFS. It has also previously been reported that the elevated CEC numbers exhibited in NSCLC patients decrease following treatment with carboplatin in combination with paclitaxel [9]. Paclitaxel and docetaxel are categorized as mitotic spindle agents with potent antiangiogenic properties [20-22]. Therefore, it seems that the baseline CEC count is a promising predictor of clinical response to the carboplatin plus paclitaxel regimen, as well as of survival. However, although several other clinical studies that have examined CECs have also found chemotherapy to be associated with either an increase or decrease in CEC number [23,24], no association was detected between gemcitabine treatment and CEC number in the pancreatic carcinoma patients in our study. Although gemcitabine has anti-angiogenic properties, higher baseline CEC levels were associated with PD in pancreatic carcinoma patients receiving gemcitabine therapy, and patients with high CEC counts exhibited poor clinical condition. It is therefore likely that the tumor type, anti-cancer drugs being administered, and the amount of time between the start of treatment and the time when CEC counts are obtained influence the number of CECs detected in cancer patients after treatment. In this study, we measured CEC levels before starting chemotherapy and at $28 \pm 7$ days after starting chemotherapy, the time of sampling might influence the changes of CEC level. Moreover, the diversity in literature regarding CEC up-or down-regulation during cancer therapy and the associated prognostic and predictive evidence might in part be explained by a differential focus on or by the lack of discrimination between these cell populations [13].

\section{Conclusions}

Although the number of patients examined in this study was small, and patients were recruited prospectively, this study, along with others, has shown the clinical importance of CEC number as a prognostic factor in advanced pancreatic carcinoma treated with gemcitabine chemotherapy, whereby high CEC counts are associated with poor prognosis. This study also found that elevated CEC counts are associated with the high expression levels of several chemokines and proangiogenic factors involved in the regulation of tumor immunological and angiogenic factors. Although this correlation between blood parameters is not proof of a causal relationship, these factors may provide viable therapeutic targets for the treatment of pancreatic carcinoma in the future. Further studies in a larger population will be required to confirm our findings.

\section{Abbreviations}

CEC: circulating endothelial cell; ECOG : Eastern Cooperative Oncology Group: CA19-9: Carbohydrate antigen 19-9; CRP: C-reactive protein; IL: Interleukin; PDGF-BB: Platelet-derived growth factor-B chain; VEGF: Vascular endothelial growth factor; HGF: Hepatocyte growth factor; PD: Progressive disease; PR: Partial response; HR: Hazard ratio; Cl: confidence interval; SD: Stable disease.

\section{Competing interests}

The authors declare that they have no competing interests.

\section{Authors' contributions}

SK and KT designed and participated in all stages of the study. SK and JH performed most of the experiments. FK and CM participated in CEC analysis, as well as the statistical analyses and discussion of the results. HU and TO recruited the patients, collected the tumor biopsy samples, and helped to draft the manuscript. All authors read and approved the final manuscript.

\section{Acknowledgments}

We thank the patients who participated in and supported this trial, as well as their families. We also thank Yuka Kitamura and Hiroko Hosoi for technical support.

This study was supported in part by a Grant-in-Aid for Cancer Research (20-9) from the Ministry of Health, Labour and Welfare. 


\section{Author details}

'Hepatobiliary and Pancreatic Oncology Division, National Cancer Center Hospital, Tokyo, Japan. ${ }^{2}$ Shien-Lab, National Cancer Center Hospital, Tokyo, Japan. ${ }^{3}$ Breast and Medical Oncology Division, National Cancer Center Hospital, Tokyo, Japan.

Received: 2 February 2012 Accepted: 18 June 2012

Published: 25 June 2012

\section{References}

1. Jemal A, Bray F, Center MM, Ferlay J, Ward E, Forman D: Global cancer statistics. CA Cancer J Clin 2011, 61:69-90.

2. Korc M: Pathways for aberrant angiogenesis in pancreatic cancer. Mol Cancer 2003, 2:8

3. Seo Y, Baba H, Fukuda T, Takashima M, Sugimachi K: High expression of vascular endothelial growth factor is associated with liver metastasis and a poor prognosis for patients with ductal pancreatic adenocarcinoma. Cancer 2000, 88(10):2239-2245.

4. Niedergethmann M, Hildenbrand R, Wostbrock B, Hartel M, Sturm JW, Richter A, Post S: High expression of vascular endothelial growth factor predicts early recurrence and poor prognosis after curative resection for ductal adenocarcinoma of the pancreas. Pancreas 2002, 25(2):122-129.

5. Hicklin DJ, Ellis LM: Role of the vascular endothelial growth factor pathway in tumor growth and angiogenesis. J Clin Oncol 2005, 23(5):1011-1027

6. Kindler HL, loka T, Richel DJ, Bennouna J, Letourneau R, Okusaka T, Funakoshi A, Furuse J, Park YS, Ohkawa S, et al: Axitinib plus gemcitabine versus placebo plus gemcitabine in patients with advanced pancreatic adenocarcinoma: a double-blind randomised phase 3 study. Lancet Oncol 2011, 12(3):256-262.

7. Blann AD, Woywodt A, Bertolini F, Bull TM, Buyon JP, Clancy RM, Haubitz M, Hebbel RP, Lip GY, Mancuso P, et al: Circulating endothelial cells. Biomarker of vascular disease. Thromb Haemost 2005, 93(2):228-235.

8. Goon PK, Lip GY, Boos CJ, Stonelake PS, Blann AD: Circulating endothelial cells, endothelial progenitor cells, and endothelial microparticles in cancer. Neoplasia 2006, 8(2):79-88.

9. Kawaishi M, Fujiwara Y, Fukui T, Kato T, Yamada K, Ohe Y, Kunitoh H, Sekine I, Yamamoto N, Nokihara H, et al: Circulating endothelial cells in non-small cell lung cancer patients treated with carboplatin and paclitaxel. J Thorac Oncol 2009, 4(2):208-213

10. Farace F, Massard C, Borghi E, Bidart JM, Soria JC: Vascular disrupting therapy-induced mobilization of circulating endothelial progenitor cells. Ann Oncol 2007, 18(8):1421-1422.

11. Bidard FC, Mathiot C, Delaloge S, Brain E, Giachetti S, de Cremoux P, Marty $M$, Pierga JY: Single circulating tumor cell detection and overall survival in nonmetastatic breast cancer. Ann Oncol 2010, 21(4):729-733.

12. Ko AH, Venook AP, Bergsland EK, Kelley RK, Korn WM, Dito E, Schillinger B, Scott J, Hwang J, Tempero MA: A phase II study of bevacizumab plus erlotinib for gemcitabine-refractory metastatic pancreatic cancer. Cancer Chemother Pharmacol 2010, 66(6):1051-1057.

13. Starlinger $P$, Brugger $P$, Reiter $C$, Schauer $D$, Sommerfeldt $S$, Tamandl $D$, Kuehrer I, Schoppmann SF, Gnant M, Brostjan C: Discrimination between circulating endothelial cells and blood cell populations with overlapping phenotype reveals distinct regulation and predictive potential in cancer therapy. Neoplasia 2011, 13(10):980-990.

14. Mancuso P, Burlini A, Pruneri G, Goldhirsch A, Martinelli G, Bertolini F: Resting and activated endothelial cells are increased in the peripheral blood of cancer patients. Blood 2001, 97(11):3658-3661.

15. Mancuso P, Calleri A, Cassi C, Gobbi A, Capillo M, Pruneri G, Martinelli G, Bertolini F: Circulating endothelial cells as a novel marker of angiogenesis. Adv Exp Med Biol 2003, 522:83-97.

16. Tartour E, Pere H, Maillere B, Terme M, Merillon N, Taieb J, Sandoval F, Quintin-Colonna F, Lacerda K, Karadimou A, et al: Angiogenesis and immunity: a bidirectional link potentially relevant for the monitoring of antiangiogenic therapy and the development of novel therapeutic combination with immunotherapy. Cancer Metastasis Rev 2011 30(1):83-95

17. Matsuo Y, Ochi N, Sawai H, Yasuda A, Takahashi H, Funahashi H, Takeyama H, Tong Z, Guha S: CXCL8/L-8 and CXCL12/SDF-1alpha co-operatively promote invasiveness and angiogenesis in pancreatic cancer. Int $J$ Cancer 2009, 124(4):853-861.
18. Kindler HL, Niedzwiecki D, Hollis D, Sutherland S, Schrag D, Hurwitz H, Innocenti F, Mulcahy MF, O'Reilly E, Wozniak TF, et al: Gemcitabine plus bevacizumab compared with gemcitabine plus placebo in patients with advanced pancreatic cancer: phase III trial of the Cancer and Leukemia Group B (CALGB 80303). J Clin Oncol 2010, 28(22):3617-3622.

19. Bertolini F, Marighetti $P$, Shaked Y: Cellular and soluble markers of tumor angiogenesis: from patient selection to the identification of the most appropriate postresistance therapy. Biochim Biophys Acta 2010, 1806(2):131-137.

20. Belotti D, Vergani V, Drudis T, Borsotti P, Pitelli MR, Viale G, Giavazzi R, Taraboletti G: The microtubule-affecting drug paclitaxel has antiangiogenic activity. Clin Cancer Res 1996, 2(11):1843-1849.

21. Hayot C, Farinelle S, De Decker R, Decaestecker C, Darro F, Kiss R, Van Damme M: In vitro pharmacological characterizations of the antiangiogenic and anti-tumor cell migration properties mediated by microtubule-affecting drugs, with special emphasis on the organization of the actin cytoskeleton. Int J Oncol 2002, 21(2):417-425.

22. Wang J, Lou P, Lesniewski R, Henkin J: Paclitaxel at ultra low concentrations inhibits angiogenesis without affecting cellular microtubule assembly. Anticancer Drugs 2003, 14(1):13-19.

23. Beaudry P, Force J, Naumov GN, Wang A, Baker CH, Ryan A, Soker S, Johnson BE, Folkman J, Heymach JV: Differential effects of vascular endothelial growth factor receptor-2 inhibitor ZD6474 on circulating endothelial progenitors and mature circulating endothelial cells: implications for use as a surrogate marker of antiangiogenic activity. Clin Cancer Res 2005, 11(9):3514-3522.

24. Hanrahan EO, Heymach JV: Vascular endothelial growth factor receptor tyrosine kinase inhibitors vandetanib (ZD6474) and AZD2171 in lung cancer. Clin Cancer Res 2007, 13(15 Pt 2):s4617-s4622.

doi:10.1186/1471-2407-12-268

Cite this article as: Kondo et al:: Circulating endothelial cells and other angiogenesis factors in pancreatic carcinoma patients receiving gemcitabine chemotherapy. BMC Cancer 2012 12:268.

\section{Submit your next manuscript to BioMed Central and take full advantage of:}

- Convenient online submission

- Thorough peer review

- No space constraints or color figure charges

- Immediate publication on acceptance

- Inclusion in PubMed, CAS, Scopus and Google Scholar

- Research which is freely available for redistribution

Submit your manuscript at www.biomedcentral.com/submit
Ciomed Central 